\title{
Médiévales
}

Langues, Textes, Histoire

75 | automne 2018

Traductions du Moyen Âge

\section{Traduire la chanson de Roland}

Translating the Song of Roland

\section{Christopher Lucken}

\section{(2) OpenEdition}

Journals

Édition électronique

URL : https://journals.openedition.org/medievales/9461

DOI : 10.4000/medievales.9461

ISSN : $1777-5892$

\section{Éditeur}

Presses universitaires de Vincennes

\section{Édition imprimée}

Date de publication : 15 octobre 2018

Pagination : 167-196

ISBN : 978-2-84292-861-2

ISSN : 0751-2708

\section{Référence électronique}

Christopher Lucken, «Traduire la chanson de Roland », Médiévales [En ligne], 75 | automne 2018, mis en ligne le 15 octobre 2019, consulté le 24 avril 2022. URL : http://journals.openedition.org/ medievales/9461; DOI : https://doi.org/10.4000/medievales.9461 
Christopher Lucken

\section{Traduire la chanson de Roland}

Le titre de la Chanson de Roland est dû à Francisque Michel, qui édita pour la première fois en 1837 le texte du manuscrit d'Oxford (Digby 23), composé ou chanté par un certain Turold et destiné à supplanter les autres versions de cette œuvre considérées désormais comme des « remaniements postérieurs ${ }^{1} \gg$. Étranger à la tradition manuscrite, ce titre peut se prévaloir du terme de chanson de geste qui désigne le genre dont cette «chanson » deviendra rapidement le principal représentant. Mais, comme le reconnaît Michel dans sa préface, il s'agit également de l'apparenter à la cantilena Rolandi qui, d'après une tradition historiographique latine et française du $\mathrm{XII}^{\mathrm{e}}$ siècle, aurait été chantée en 1066 par un certain Taillefer lors de la bataille de Hastings afin de stimuler l'ardeur au combat des troupes de Guillaume le Conquérant. Cette cantilène apparaissait en effet comme l'expression emblématique d'une poésie « primitive » étrangère à la tradition classique qui dominait la littérature française ${ }^{2}$. « Les Barbares avaient la passion de la musique et des vers », affirme par exemple Chateaubriand en 1831 dans la sixième de ses Études ou discours historiques sur la chute de l'empire romain, la naissance et les progrès du christianisme, et l'invasion des barbares, consacrée aux «Mœurs des barbares » et en particulier à leur production poétique ; « leur muse s'éveillait aux combats, aux festins et

1. La Chanson de Roland ou de Roncevaux, publiée pour la première fois par F. MichEL, Paris, 1837, préface, p. XI-XIV et XVI. $C f$. A. TAYLOR, «Was there a Song of Roland ? , Speculum, 76 (2001), p. 28-65 ; C. LuCKEN, « De la "chanson" de Roland au manuscrit d'Oxford. En quête d'un chant primitif », dans C. Cazanave éd., Mémoire épique et Génie du lieu, Bien Dire et Bien Aprandre, hors série, $\mathrm{n}^{\circ} 2$ (2017), p. 141-164.

2. Cf. P. VAn Tieghem, « La notion de vraie poésie dans le préromantisme européen », dans ID., Le Préromantisme. Études d'histoire littéraire européenne, Paris, 1924, t. I, p. 1771 ; C. LuCKEN, " "Ainsi chantaient quarante mille Barbares". La vocation de la poésie barbare chez les romantiques français », dans J. Rigoli et C. CARuso éd., Poetiche barbare - Poétiques barbares, Ravenne, 1998, p. 153-181. 
aux funérailles », précise-t-il en se référant au De Germania de Tacite. "Plusieurs siècles après la conquête de l'empire romain », poursuit Chateaubriand, «l'usage des hymnes guerriers continua : les défaites amenaient des complaintes latines dont l'air est quelquefois noté dans les vieux manuscrits ». Enfin, « ces rythmes militaires se viennent terminer à la chanson de Roland, qui fut comme le dernier chant de l'Europe barbare ${ }^{3} »$. $\mathrm{La}$ « chanson de Roland » dont il est question ici n'est pas celle que nous désignons désormais sous ce nom, mais la cantilena Rolandi.

La découverte de cette «ballade hérö̈que » devait notamment permettre de pourvoir la littérature - et la nation - françaises d'un chant épique analogue aux poèmes d'Ossian publiés en 1760 et 1765 par Macpherson, ou aux Volkslieder - aux Chants du peuple - publiés en 1778 et 1779 par Herder. Les deux ou trois poèmes consacrés à la bataille de Roncevaux qu'on connaissait alors ne s'apparentaient guère à un chant guerrier. La version du manuscrit d'Oxford semblait en revanche pouvoir y correspondre. Le titre qui lui fut donné était chargé en tout cas de le suggérer.

Francisque Michel n'a pas accompagné son édition de la Chanson de Roland d'une traduction. Il s'est contenté d'un index et de gloses marginales traduisant les mots ou les expressions qui lui paraissaient les plus difficiles à comprendre. Comme le note toutefois Étienne Jean Delécluze, qui en donna en 1845 la première traduction, «ce livre [ayant] été écrit dans les premières décades du douzième siècle », sa " lecture en est trop difficile pour qu'elle laisse saisir tout à la fois les détails, en suivant rapidement la marche de l'action ${ }^{4} »$. L'affirme à son tour, en 1852, Louis (ou Ludovic) Vitet : si l'édition de Michel ne semblait rien laisser à désirer, « son travail n'en était pas moins incomplet par cela seul qu'il s'adressait uniquement aux savans. Le public, en pareille matière, a droit de n'être pas oublié. Pour lui donner la clé d'une telle œuvre, il ne suffisait pas d'un glossaire expliquant à peine quelques mots ; c'est une traduction qu'il fallait ${ }^{5} \gg$. Comment traduire cependant un tel chant ?

Habituées aux " belles infidèles ${ }^{6}$ », les traductions françaises ne cherchaient guère à respecter les propriétés singulières du texte original. Tandis qu'en Allemagne on tendait à privilégier la notion de fidélité, « d'autres nations », affirme August Wilhelm Schlegel vers 1830, « ont adopté en poésie une phraséologie complètement conventionnelle, si bien qu'il est purement impossible de traduire poétiquement dans leur langue, comme par exemple

3. Chateaubriand, Études ou discours historiques sur la chute de l'empire romain, la naissance et les progrès du christianisme, et l'invasion des barbares, Paris, 1831, p. 426, 432 et 433 .

4. Roland ou la chevalerie, trad. E. J. Delécluze, Paris, 1845, t. I, préface, p. XV.

5. L. Vitet, « La Chanson de Roland », Revue des Deux Mondes, 14 (1852), p. 817-864 (cit. p. 822 ; voir aussi p. 827).

6. Cf. R. ZuBER, Les « Belles infidèles » et la formation du goût classique [1968], Paris, 1995. 
en français. [...] C'est comme s'ils désiraient que chaque étranger, chez eux, doive se conduire et s'habiller d'après leurs mœurs, ce qui entraîne qu'ils ne connaissent à proprement parler jamais d'étranger ${ }^{7}$ ». " S'il y a quelque mérite à traduire », estimait par exemple Collardeau à la fin du XVIII ${ }^{e}$ siècle, «ce ne peut être que celui de perfectionner, s'il est possible, son original, de l'embellir, de se l'approprier, de lui donner un air national et de naturaliser, en quelque sorte, cette plante étrangère ${ }^{8} »$. On était enfin parvenu à découvrir « la version la plus ancienne » de la Chanson de Roland, que l'on avait éditée en respectant autant que possible le texte original de ce témoin primitif de « notre ancienne littérature ${ }^{9} » ;$ « l'antiquité de son langage » semblait prouver qu'elle avait été chantée à Hastings, qu'elle était apparentée aux hymnes guerriers des Germains dans lesquels, selon Chateaubriand citant Tacite, « l'oreille dédaigneuse des Grecs et des Romains n'entendaient [...] que des croassements de corbeaux ou des sons non articulés, sans aucun rapport avec la voix humaine »; elle semblait posséder l' « énergie » et la « férocité » des chants barbares susceptibles d'entraîner ceux qui les entendent à se jeter contre l'ennemi et à se battre jusqu'à la mort ${ }^{10}$, ou encore « la simplicité, la brusquerie, l'énergie » propres à « l'épopée populaire ${ }^{11}$ ». Comment donc pouvait-on vouloir « perfectionner », « embellir » et « naturaliser » cette œuvre, et la priver ainsi de son étrangeté et de sa véritable nature pour la conformer aux normes esthétiques de la poétique classique qui dominait alors la littérature française ? Lui imposer les règles d'une langue policée dont on valorisait l'ordre et la clarté, qui était la plus à même d'incarner la logique de la raison et qui semblait privilégier la prose ${ }^{12}$, ne pouvait qu'altérer les traits qui faisaient de la Chanson de Roland l'expression d'une poésie primitive authentique.

Depuis la publication du texte du manuscrit d'Oxford, plus de cinquante traductions ou adaptations en ont été proposées, soit en moyenne une tous les trois ou quatre ans ${ }^{13}$. Je m'en tiendrai ici à la période qui va de la

7. A. W. Schlegel, Geschichte der klassischen Literatur, cité d'après A. BeRman, L'Épreuve de l'étranger. Culture et traduction dans l'Allemagne romantique, Paris, 1984, p. 62.

8. Cité d'après A. Berman, L'Épreuve de l'étranger..., p. 62, n. 2.

9. F. Michel, La Chanson de Roland ou de Roncevaux..., p. XII, XVII et XVIII.

10. Chateaubriand, Études ou discours historiques..., p. 428 et 433.

11. Alors qu'il ne connaissait pas encore le texte du m. d'Oxford et se fondait pour sa Dissertation sur le Roman de Roncevaux publiée à Paris en 1832 sur la version du ms. conservé désormais à Châteauroux, H. Monin doutait que cette œuvre soit une véritable « épopée populaire »; il estimait néanmoins qu'elle réunissait « souvent les caractères de cette poésie : je veux dire la simplicité, la brusquerie, l'énergie ; de plus, la répétition continuelle des mêmes épithètes, des mêmes expressions, des mêmes vers. Il y a peu d'ouvrages qui ressemblent davantage sur tous ces points aux poésies populaires des divers peuples d'Europe » (H. Monin, Dissertation sur le Roman de Roncevaux, Paris, 1832, p. 69).

12. Cf. Rivarol, L'Universalité de la langue française [1783], Paris, 1998, en particulier p. 72-73 et 76-77.

13. Cf. M. LÉONARD, « La mort de Roland. La traduction a-t-elle su restituer l'émotion ? », dans A. Corbellari et A. Schnyder éd., Translatio litterarum ad penates. Das Mittelalter 
première traduction à celle de Joseph Bédier, parue en 1922. Sur les dix-huit traductions complètes qui précédèrent cette dernière, onze sont en vers ou en prose rythmée, soit plus de la moitié. C'est à elles que je m'intéresserai principalement ici dans la mesure où elles s'efforcent de répondre d'une manière ou d'une autre au titre donné à cette œuvre et proposent différentes solutions pour en restituer le caractère lyrique. Il ne m'est pas possible en revanche de m'arrêter sur les traductions en prose et je me contenterai de citer celles qui sont susceptibles d'éclairer mon propos. Je n'ai pas davantage la place d'analyser les différentes réflexions sur la traduction qui ont pu influencer les traducteurs de la Chanson de Roland et de comparer leurs entreprises avec ce qui a été fait pour des œuvres analogues, par exemple pour les poèmes d'Ossian ${ }^{14}$. Je ne pourrai pas non plus étudier les traductions elles-mêmes et devrai me contenter de présenter ce qu'en disent leurs auteurs dans les préfaces qui accompagnent leurs publications.

S'appuyant sur le célèbre chapitre de la Deffense et illustration de la langue françoyse de Du Bellay intitulé « De ne traduire les poètes » (1549), Bédier estime quant à lui que « tout traducteur dissocie nécessairement et détruit » la « convenance de l'idée et du sentiment au rythme et au nombre de la phrase, au son, à la couleur et à la saveur des mots », en quoi réside « l'art d'écrire », qu'il « est l'esclave de la littéralité et qu'il peut bien rendre en son propre langage la pensée, mais non la musique de la pensée, non cette petite chose, le style ${ }^{15}$. Plutôt que de s'efforcer de restituer sans pouvoir y parvenir la musique de cette chanson en la traduisant en vers, Bédier ne prétend - ditil - qu'à « l'exactitude littérale ». Malgré les remontrances d'Henri Chamard qui s'employa à réviser son travail, il s'en est donc tenu à une traduction en " $\operatorname{prose}^{16}$ ». Après avoir cherché à préciser ce qui a poussé la plupart de ses premiers traducteurs à vouloir en restituer le chant, de Francis Génin en 1850 à Chamard en 1919, j'analyserai donc pour finir les motifs qui ont amené Bédier à y renoncer et les enjeux d'un tel refus. Sa décision a opéré en effet un véritable tournant dans l'histoire des traductions de la Chanson de Roland. $\mathrm{Si}$ quelques traductions en vers verront encore le jour par la suite, comme celle de Fagus en $1929^{17}$ ou celles de Raoul Mortier en $1930^{18}$ et en $1936^{19}$, c'est

übersetzen - Traduire le Moyen Âge, Cahiers du Centre de traduction littéraire de Lausanne, 47 (2005), p. 193-219.

14. Cf. P. Van Tieghem, Ossian en France, Paris, 1917 ; ID, « Ossian et l'ossianisme au XVIII ${ }^{e}$ siècle », dans Id., Le Préromantisme..., p. 195-287 (principalement p. 224-239).

15. La Chanson de Roland, publiée d'après le manuscrit d'Oxford et traduite par J. BÉDIER, Paris, 1922, p. XIV-XV (je souligne).

16. Ibid., p. XIV.

17. La Chanson de Roland, publiée dans l'original et transcrite en vers par FAGUs, Paris, 1929.

18. La Chanson de Roland, traduction de R. Mortier, Paris, 1930.

19. La Chanson de Roland, d'après le manuscrit d'Oxford, avec une traduction assonancée, par R. MORTIER, Paris, 1936, 1940. 
presque toujours en prose (serait-elle découpée en suivant le vers du texte original) que l'on rendra désormais cette œuvre. Ce n'est qu'au xxI ${ }^{\mathrm{e}}$ siècle - dans la foulée des travaux qui ont été consacrés à la poésie orale, en particulier ceux de Paul Zumthor - qu'on tentera à nouveau de la traduire en vers, avec Jean-Louis Paul ${ }^{20}$, François Regnault et Bertrand Suárez-Pazos ${ }^{21}$, et enfin Francis Boyer ${ }^{22}$.

\section{Où trouver la règle pour rétablir une musique évanouie?}

Alors que la première traduction de la Chanson de Roland, composée en prose par Delécluze, cherchait avant tout à en faire connaître le sens général ${ }^{23}$, François Génin s'est efforcé de produire en regard de sa nouvelle édition publiée en 1850 une traduction qui puisse rendre compte de la musique de cette chanson de geste ${ }^{24}$. « Mais où trouver aujourd'hui la règle pour rétablir cette musique évanouie ? »demande-t-il dans son introduction ${ }^{25}$. Cette question porte en fait sur la prononciation, confrontée à une orthographe et à une versification qui paraissent irrégulières. Mais elle aurait tout aussi bien pu porter sur la traduction de cette œuvre. La règle que Génin s'est donnée pour tenter d'en rendre la « musique évanouie » a été de « traduire aussi peu que possible » en utilisant « une langue chargée d'archaïsme » et « une prose cadencée et rythmée, du vers blanc ${ }^{26}$. D'une part, comme il ne lui semblait pas « possible de traduire fidèlement une composition $\mathrm{du} \mathrm{XI}^{\mathrm{e}}$ siècle dans la langue académique du XIX ${ }^{\mathrm{e}}$ siècle », il décida d' "employer la langue si riche, flexible et colorée du $\mathrm{XVI}^{\mathrm{e}}$ siècle », une sorte d'état intermédiaire si l'on veut, se donnant notamment comme modèle la langue utilisée par Amyot dans ses traductions ${ }^{27}$. D'autre part, comme « la prose, telle qu'on la parle dans les relations les plus communes de la vie, la vile prose ne paraissait point alors un assez digne instrument littéraire », et que « la difficulté d'un mètre constant et d'une rime obligée faisait obstacle à la fidélité et à l'exactitude du traducteur », Génin a trouvé ici aussi « un moyen terme »: soit une « prose cadencée et rythmée », ou «vers blanc» (dont il varie toutefois la longueur), vers métrique non rimé qui a bénéficié d'un certain succès avec le

20. La Chanson de Roland, trad. J.-L. PAUL, Paris, 2004.

21. Traduction en décasyllabes assonancés qui fut récitée par des comédiens le 20 mars 2005 à l'auditorium du Louvre (Paris), dans une mise en espace de Brigitte Jaques-Wajeman, mais qui n'a pas été publiée.

22. F. BOyer, Rappeler Roland. Rappeler Roland, Chanson de Roland (nouvelle traduction), Cahier Roland, Paris, 2013, p. 81-257.

23. $C f$. E. J. DelÉcluze, préface à Roland ou la chevalerie..., t. I, p. XVI et XXII-XXIII.

24. La Chanson de Roland, poème de Théroulde, texte critique accompagné d'une traduction et de notes par F. GÉnIN, Paris, 1850.

25. Ibid., p. CLXVI.

26. Ibid., p. CLXVIII.

27. Ibid. 
romantisme, en particulier pour traduire les vers blancs des poètes anglais et allemands ${ }^{28}$, et dont la légitimité paraissait d'autant plus grande que Génin croyait, en s'appuyant sur une mauvaise lecture de la version en prose des Quatre livres des Rois réalisée au XII ${ }^{\mathrm{e}}$ siècle, que ce vers avait été appliqué « aux traductions dès l'origine de notre littérature ${ }^{29}$ ».

L'édition et la traduction de Génin suscitèrent de nombreuses réserves. Dans un long compte rendu particulièrement acerbe, Paulin Paris s'en prend aussi bien à sa langue qu'à son emploi du vers blanc ${ }^{30}$ :

Je crois avoir déjà dit que M. François Génin avait choisi, pour traduire un poëme écrit en vers réguliers de dix syllabes assonantes, un système particulier de vers blancs. Ses vers ne sont pas toujours faciles à reconnaître ; mais il est au moins certain que sa traduction, annoncée comme faite dans la langue du seizième siècle, n'appartient au français d'aucune époque. C'est un mélange baroque de mots malsonnants et d'inversions bizarres, qui porte nécessairement sur les nerfs les plus robustes. Quelle singulière fantaisie, en effet ! Choisir l'époque la plus gourmée de notre littérature pour l'appliquer à la traduction d'un poëme des temps primitifs ! Et comme si cela ne suffisait pas, disposer ces mots surannés en prose cadencée, en vers blancs, les vers blancs ! dont nous avons horreur en France, notre prosodie n'étant pas assez compliquée pour se passer de la difficulté de l'assonance ou de la rime. M. Génin ne s'est pas contenté de supprimer la rime ; il a fait un pêle-mêle de toutes les mesures, et préparé pour notre oreille et nos yeux le plus abominable mélange de tons et de couleurs dont peintre d'enseigne ait jamais eu la coupable pensée. Mon Dieu, quels tristes efforts pour singer l'originalité !

Ludovic Vitet sera moins sévère : «M. Génin excelle dans cet art malaisé de serrer de près son modèle, d'en reproduire exactement le tout, l'allure et l'esprit ${ }^{31}$.»Vitet ne semble pas gêné par le « vers blanc » employé par Génin. Il regrette toutefois que ce dernier ait préféré à « notre langue d'aujourd'hui » celle du XvI e siècle. Certes, concède-t-il, « qu'il ait habilement tenu cette gageure, nous ne le contestons pas, nous reconnaissons même qu'il $\mathrm{y}$ a dans ce vieux langage plus de ressources que dans notre moderne idiome pour rendre avec vérité le français du $\mathrm{XI}^{\mathrm{e}}$ siècle ». Mais, poursuit-il ${ }^{32}$ :

Pour qui sont faites les traductions ? est-ce pour ceux qui peuvent s'en passer? Quand on lit couramment les écrits d'Amyot, n'est-on pas en état de comprendre, sans trop d'efforts, la chanson de Roland ? C'est donc, à vrai dire, pour lui seul, pour son propre plaisir, que M. Génin a fait sa

28. $C f$. en particulier C. LomBEz, « La traduction poétique et le vers français au $\mathrm{XIX}^{\mathrm{e}}$ siècle », Romantisme, 140 (2008), p. 99-110.

29. F. GÉnIN, La Chanson de Roland, p. CLXIX ( $c f$. aussi p. CLXXII).

30. P. PARIs, "La Chanson de Roland (édition de M. Génin) (premier article) », Bibliothèque de l'École des chartes, 12 (1851), p. 297-338 (cit. p. 337-338).

31. L. Vitet, « La Chanson de Roland »..., p. 827.

32. Ibid., p. 827-828. 
traduction. Il a moins songé à son lecteur qu'à sa fantaisie d'antiquaire ; être compris n'a pas été souci principal. Aussi quand par hasard il rencontre dans son texte un terme encore intelligible, un terme qui n'a pas vieilli, au lieu de le conserver, il s'amuse à en choisir un autre obscur et hors d'usage ; c'est ainsi, par exemple, qu'il traduit ces mots : en tel bataille, par ceux-ci : en tel estrif. N'est-il pas évident que cette manière d'éclaircir un texte est peu secourable aux ignorans ? L'inconvénient radical d'une telle traduction, c'est qu'elle a besoin d'être traduite.

Aussi, tandis que Génin a cherché à rendre la «musique évanouie » de cette chanson (même si, comme Paulin Paris, on peut ne pas être convaincu par sa tentative), Vitet estime qu'il faut plutôt en fournir une « traduction claire et fidèle » qui puisse « être compris[e] » de ses lecteurs comme de tous ceux qui souhaitent connaître l'histoire de Roland : car « comment parler d'une œuvre que personne n'a lue, que personne ne peut lire, hormis quelques savans ? ${ }^{33}$. Vitet proposera donc une traduction en prose, se permettant en outre de supprimer de nombreuses répétitions et d'abréger le texte comme s'il s'agissait d'en offrir une simple analyse ${ }^{34}$.

Dans l' «Aperçu » qui ouvre sa traduction de la Chanson de Roland publiée en 1861, Pierre Jônain reconnaît lui aussi certaines qualités à la traduction de Génin (qu'il qualifie toutefois de prose). Mais si ce dernier « a fait heureusement arriver le Roland jusqu'à notre seizième siècle », Jônain affirme que « le vif désir que ce poème national soit plus généralement connu de la nation » lui a donné « l'audace d'essayer à la faire parvenir au dix-neuvième, non sans archaïsmes encore, mais en lui rendant la mesure et plus que l'assonance des vers ${ }^{35}$. Contrairement à Vitet et « en opposition à de très-doctes et honorés Critiques », il estime « que les poésies doivent être traduites en vers, et même plusieurs fois, successivement, à mesure que les langues vivantes se transforment et se modifient » :

Les grands poèmes sont des fleuves qu'il faut détourner sur notre sol avec leur rythme de cours et de murmure ; des tableaux dont nous voulons des copies qui reproduisent tout le dessin, tout le clair obscur et qui même rajeunissent les couleurs ${ }^{36}$.

En revanche, soutient Jônain en poursuivant la comparaison (classique) entre la traduction et la copie d'une peinture, « les mot à mot, les interprétations, les translations en prose ne sont que de froids décalques ou de pâles gravures ${ }^{37} \gg$. Jônain s'inscrit dans une tradition remontant à Cicéron,

33. Ibid.

34. Ibid., p. 828-829.

35. Roland, poème hérö̈que de Théroulde, trouvère du XIe siècle, traduction en vers français par P. JônAIN, sur le texte et la version en prose de F. Génin, Paris, 1861, p. XI.

36. Ibid., p. XI-XII.

37. Ibid., p. XII. 
qui affirme dans son De optimo genere oratorum qu'il faut traduire « non en interprète, mais en orateur », et, plutôt que de rendre le texte original «mot pour mot » (verbum pro verbo), qu'il est préférable de l'adapter à la langue cible en restituant le «poids » ou le style des mots (comme du texte dans son ensemble) ${ }^{38}$. Une traduction littérale se contente en effet de rendre les idées contenues dans chaque mot, et non la «forme » du poème, alors que c'est elle qu'il « faut à tout prix s'efforcer de [...] reproduire ». Car, poursuit Jônain qui semble répondre à Vitet souhaitant que l'on puisse «parler» de cette chanson, « on ne parle pas la poésie, on la chante $»^{39}$. Jônain traduira donc la Chanson de Roland en laisses de décasyllabes pourvus de rimes qu'il essaye d'enchaîner deux par deux, sous forme de rimes plates, embrassées ou croisées, selon les cas, avec de temps à autre un vers surnuméraire qui vient se greffer comme il peut sur les autres ${ }^{40}$.

Dans les «Quelques mots sur cette nouvelle traduction » par lesquelles il conclut l'introduction de son ouvrage paru en 1865, Adolphe d'Avril commence à son tour par justifier la traduction de la Chanson de Roland en invoquant la difficulté de sa langue, qui n'est compréhensible que par ceux qui en ont fait une «étude spéciale » et ne peut donc être abordée «que par un petit nombre d'élus », et sa volonté d'en assurer la « popularité »: «Il fallait donc traduire la Chanson de Roland ${ }^{41}$. » Connaissant les traductions en prose (ou en vers blancs) de Delécluze, Génin, Vitet et Alexandre de Saint-Albain ${ }^{42}$, et ce qui était alors la seule traduction en vers rimés, due à Jônain, d'Avril affirme avoir travaillé « d'après un autre système » :

Le but que je me suis proposé [...] est de vulgariser les précieux restes de nos épopées nationales sans en altérer les traits, sans leur ôter la couleur, sans en abaisser le ton, c'est-à-dire en leur laissant la vie. Je n'ai pas cherché à refaire la Chanson de Roland: on ne refait pas à une époque le poëme d'une autre époque. Ce que j'ai essayé c'est de reproduire plutôt que de traduire ; c'est de conserver le style de Théroulde. Pour atteindre ce but, il fallait s'appliquer à ne pas altérer la forme. On ne pouvait pas se permettre d'ajouter un seul ornement ni de changer l'allure du texte original. Je n'ai donc rien ajouté et j'ai modifié le moins possible ${ }^{43}$.

38. Cić́ron, De optimo genere oratorum, V, 14, éd. et trad. A. Yon, dans L'Orateur. Du meilleur genre d'orateurs, Paris, 1964, p. 114.

39. P. JônaIN, Roland, poème hérö̈que de Théroulde ..., p. XII-XIII.

40. Précisons cependant que, si Jônain choisit de traduire cette chanson « presque vers pour vers », il estime nécessaire en revanche - un peu comme Vitet cette fois - de «l'abréger» : cf. ibid., p. XIII-XIV.

41. La Chanson de Roland, traduction nouvelle, avec une introduction et des notes, par A. D’AvriL, Paris, 1865, p. CXXVII-CXXVIII.

42. La Chanson de Roland. Poëme de Théroulde, suivi de la Chronique de Turpin, traduction d'A. DE SAINT-Albin, Paris, 1865.

43. A. D'AvriL, La Chanson de Roland, p. CXXVIII. 
Alors qu'il entend conserver le style et la forme particulière de cette chanson et en offrir une traduction aussi fidèle que possible, d'Avril a cependant « cru devoir sacrifier l'assonance ». Celle-ci, affirme-t-il, « dit quelque chose à l'oreille, et [...] a de la valeur dans les œuvres chantées, mais [...] ne parle pas beaucoup aux yeux ». D'Avril n'a pas tenté pour autant d'y « ajouter la rime », car « il aurait fallu modifier le texte, et l'on n'aurait pu le faire sans altérer la forme, sans compromettre le ton épique de Théroulde », " reconnaissant avec les auteurs de l'Histoire littéraire de la France qu'en voulant remplacer les assonances par des rimes exactes, les trouvères postérieurs ont corrompu le caractère et altéré le style de la composition primitive telle qu'elle se trouve dans le manuscrit d'Oxford ». Il a conservé en revanche le « vers de dix pieds », « parce que c'est le vers même de Théroulde » et qu'il lui « paraît préférable à tous les autres pour la gravité et la vivacité du récit épique », d'autant qu'il est «plus libre » et ne présente «pas les mêmes difficultés » que l'alexandrin. Sa traduction est donc faite de « vers blancs de dix pieds». Si d'Avril n'a pu faire « sentir la versification par la rime », il s'est imposé « la règle de placer les accents [...] là où Théroulde les a placés, et comme l'usage l'a généralement consacré, c'est-à-dire à la quatrième syllabe et à la dixième », soit à l'hémistiche et à la fin du vers. Il s'est appliqué « à éviter l'hyatus » («pour respecter les habitudes des oreilles françaises ») et a « exclu l'enjambement qui est une innovation et que nos anciens poëtes n'admettaient pas parce qu'il déplace l'accent, cet élément essentiel de la versification française ». En revanche, il n'a pas « rejeté absolument l'inversion qui se trouve dans nos poëmes primitifs ». En ce qui concerne le lexique, il «a évité autant que possible les expressions et les tournures archaïques et croit n'avoir conservé aucun mot qui ne soit intelligible ». Il n'a pas non plus cherché «à faire de la poésie naïve et se donner les airs du moyen âge, en supprimant quelques articles et pronoms », et ne l'a fait qu'à « son vers défendant », omettant parfois aussi pour les mêmes raisons « la négative pas ou la négative $n e »^{44}$. D'Avril s'est donc efforcé de respecter dans une certaine mesure la prosodie et la syntaxe du texte original tout en modernisant la langue et la versification conformément à l'usage contemporain.

Si d'Avril a choisi de conserver le décasyllabe, c'est l'alexandrin qu'emploiera Alfred Lehugeur pour traduire en 1870 la Chanson de Roland en « vers modernes ${ }^{45}$ ». Affirmant répondre au vœu de Vitet qui souhaitait « une traduction claire et fidèle », il a tenté - dit-il - d' « observer envers l'original une fidélité plus grande » que ce dernier en choisissant de «le traduire en vers $\gg^{46}$ :

44. Ibid., p. CXXVIII-CXXXI.

45. La Chanson de Roland, poème français du Moyen Âge, traduit en vers modernes par A. Lehugeur, Paris, 1870.

46. Ibid., p. XIV. 
Il m'a semblé que, si les mots sont plus respectés par la prose, les vers conservent mieux l'esprit, l'allure, le ton, et que, dans une œuvre dont le prix tient beaucoup moins à l'expression qu'à la pensée, il fallait revêtir celle-ci de la forme qui peut lui donner le plus d'avantage et d'éclat, sans sacrifier l'exactitude.

Pour y parvenir, estime Lehugeur, il faut « un vers ferme et concis, sonore à l'occasion, mais simple et sans apprêt », qui serve de « moule » où « couler chaque vers du vieil auteur »; car, précise-t-il, «à bien peu d'exceptions près, c'est vers pour vers [qu'il s'est] appliqué à la rendre ». Et si on demande pourquoi il a « remplacé le vers de dix syllabes par l'alexandrin, c'est d'abord que le français d'aujourd'hui, moins serré que celui du temps de Guillaume [le Conquérant], exige, pour se mouvoir, un peu plus d'espace » : il aurait donc été difficile autrement de traduire « vers pour vers ». Mais, poursuit Lehugeur, « c'est surtout parce que le mètre primitivement employé pour les romans héroïques, et abandonné dès la fin du XII siècle, est loin de posséder au même degré que l'autre le son noble et grave qui convient à l'épopée ${ }^{47}$. Comme l'écrit Georges Lhote dans son Histoire du vers français ${ }^{48}$ :

Il est de tradition que le poème épique soit écrit en vers et que l'auteur y emploie un mètre majestueux, digne de la grandeur et du sujet qu'il traite. Virgile avait usé de l'hexamètre. En France, depuis l'échec de Ronsard [dont la Franciade était composée comme les chansons de gestes traditionnelles en décasyllabes], il convient de recourir à l'alexandrin et, de toutes les épopées composées au XVII ${ }^{\mathrm{e}}$ siècle, il n'y en a pas une seule dont l'auteur n'ait pas respecté cette règle, à laquelle Voltaire n'a pas manqué de se soumettre.

Si l'emploi de ce «vers hérö̈que », le plus « noble » et le plus prestigieux de la prosodie française, éloigne la Chanson de Roland de la chanson de geste médiévale, il permet en revanche de le rattacher à l'épopée classique.

Dans sa monumentale édition de la Chanson de Roland publiée en 1872, Léon Gautier affirme avoir dû choisir pour sa traduction « entre deux systèmes » : la «simple prose » employée par Génin et Saint-Albin, et les «vers rimés » employés par Jônain et Lehugeur ${ }^{49}$. Il avoue avoir été « séduit » tout d'abord par la solution intermédiaire adoptée par d'Avril. Mais, poursuit-il, si le « vers blanc » est «très-favorable au sentiment du rythme », il ne le serait

47. Ibid., p. XV.

48. G. LotE, Histoire du vers français, t. IX, Troisième partie : le XVIII siècle, III, Les genres et les formes. La versification. Du classicisme au romantisme, texte revu par J. TAMINE, A. Niklas-S Alminen, L. Victor, Aix-en-Provence, 1996, p. 8.

49. La Chanson de Roland, texte critique accompagné d'une traduction nouvelle et précédé d'une introduction historique, par L. GAUTIER, Tours, 1872, t. I, p. CXCVI. Sur l'importance de la Chanson de Roland pour Gautier, $c f$. C. LuCKEN, «Actualité de la Chanson de Roland. Une "chanson populaire" au programme d'agrégation », dans V. CANGEMI, A. Corbellari, U. Bähler éd., Le Savant dans les lettres, Rennes, 2014, p. 93-106. 
pas « à l'exactitude de la couleur. Or, affirme Gautier, la couleur, c'est le seul style du Roland ». Mise en avant dans les années 1820-1830 sous l'influence des romans historiques de Walter Scott par des historiens « narrativistes » comme Augustin Thierry et Prosper de Brabante, la notion de « couleur » ou de « couleur locale » désigne la capacité de restituer d'une manière à la fois vivante et fidèle la période décrite, à la manière d'un tableau, au lieu de s'en tenir à une étude analytique, et l'emploi privilégié pour ce faire d'un style narratif proche de ce qu'offre la littérature ${ }^{50}$. La «couleur» de la Chanson de Roland désigne ainsi sa propension à nous donner à voir l'histoire qu'elle raconte. D'ailleurs, affirme encore Gautier, « il est tel vers qu'on traduit plus exactement en vingt syllabes qu'en dix ; tel équivalent est plus vrai que tel mot servile. Nous avons donc conservé le principe excellent de la traduction vers par vers ; mais nous n'avons pas voulu de ce lit de Procuste qu'on appelle un vers ». Gautier reconnaît néanmoins au vers une certaine unité ; c'est pourquoi il traduit «vers par vers » plutôt que de suivre le cours de la prose sans marquer de coupures comme l'avaient fait Génin, Vitet et Saint-Albin. Mais le vers apparaît davantage comme une unité sémantique ou syntaxique que comme une mesure fondée sur le nombre de syllabes et un rythme particulier.

Si c'est à la peinture que Gautier apparente la Chanson de Roland, c'est à la musique que Louis Petit de Julleville entend avant tout l'associer dans sa traduction publiée en 1878. " Il faut toujours se souvenir », affirme-t-il après avoir traité de la versification de cette chanson et avant de parler de sa traduction, « que nos chansons de geste étaient chantées avec un accompagnement de violon, qu'on appelait vielle. Les procédés du musicien ont dû influer sur ceux du poète ${ }^{51}$. » Avant de présenter son propre travail, Petit de Julleville va analyser les sept traductions qui précèdent en soulignant ce qui lui paraît en être les qualités et les défauts ${ }^{52}$. Il les classe pour cela en quatre systèmes. D'une part :

MM. Delécluze, de Saint-Albin et Léon Gautier ont traduit en prose ; ce qui permet d'être très-fidèle au sens littéral, beaucoup plus même que ne l'ont été en général nos traducteurs. Nul d'eux n'est tout à fait exempt de paraphrase. Mais le grand inconvénient qu'il y a à traduire un poëte en prose, c'est que le traducteur ne peut rien conserver de ce qui constitue proprement la poésie de son texte. Il rendra le sens, la grandeur des pensées et des sentiments, la vivacité de quelques inventions, la vigueur de quelques peintures, mais la poésie lui échappe nécessairement, puisqu'il écrit en prose.

50. Cf. J. KAMERBEeK, Tenants et aboutissants de la notion de couleur locale, Utrecht, 1962 ; C. FluCKIGER, L'Histoire entre art et science : la « couleur locale » chez Thierry et Barante, mémoire de licence, Université de Genève, 1995.

51. La Chanson de Roland, traduction nouvelle rythmée et assonancée. Avec une introduction et des notes, par L. Petit de Julleville, Paris, 1878, p. 88.

52. Ibid., p. 88-91. 
Le vers serait indissociable de la poésie : celle-ci ne saurait donc faire l'objet d'une traduction en prose.

Le deuxième système retenu par Petit de Julleville est celui de Génin (dont il critique à son tour la langue archaïque) :

Pour éviter ce reproche [d'avoir traduit en prose], il a traduit Roland dans une sorte de prose rhythmée, en vers blancs de huit, dix, douze syllabes, qui se succèdent sans règle et sont même alignés comme la simple prose ; parfois une ligne de prose intervient au milieu de ce rythme capricieux, quand le traducteur n'a pas pu trouver la mesure nécessaire. Ce procédé singulier berce agréablement l'oreille de l'auditeur, mais ne rend à aucun degré le mouvement de l'original.

Le troisième système est celui de Jônain et Lehugeur, qui ont traduit en « vers rimés » :

M. Jônain a choisi le vers de dix syllabes ; M. Le Hugeur, l'alexandrin. Il me paraît impossible, dans un tel système de traduction, d'être exact, sans risquer d'être plat; surtout si on emploie le vers de dix pieds, qui est celui de l'original, comment rendre tous les mots, et amener cependant la rime à la fin du vers ? M. Le Hugeur s'est donné un peu plus d'espace et de facilité en choisissant l'alexandrin ; mais il change ainsi d'un bout à l'autre le rythme du poëme ; et je n'aime guère la façon dont il s'en justifie en disant que « le mètre employé primitivement pour les romans héroïques, et abandonné dès la fin du douzième siècle, est loin de posséder au même degré que l'autre (l'alexandrin) le son noble et grave qui convient à l'épopée ». Notre affaire est de traduire et non de corriger les originaux.

Petit de Julleville estime plus largement qu'en « introduisant dans la traduction la rime, ou plate ou croisée, on y mêle un élément que l'original n'a point connu ; il n'a connu que l'assonance ». S'il admet qu'il est impossible de traduire Homère ou Virgile en conservant « la versification de l'original », il n'en serait pas de même pour Roland car il « est écrit en français, et le système de versification du poëme, s'il n'est plus usité, reste toujours applicable dans le français moderne. Dès lors pourquoi n'essayer point de le conserver? »

Le quatrième système est celui que « tenta » de suivre d'Avril dans sa « remarquable traduction » :

Elle est en vers blancs, c'est-à-dire non rimés, décasyllabiques, et elle rend l'original vers pour vers, et le plus possible, mot pour mot. Je ne reprocherai qu'une chose à M. d'Avril, c'est de n'avoir pas été assez loin dans son propre système. En s'astreignant à observer dans la mesure de ses vers les règles de la versification moderne, M. d'Avril s'est imposé une inutile et gênante entrave. Beaucoup de vers du Roland, qu'il aurait pu rendre en transcrivant mot pour mot l'original, ont dû être modifiés pour qu'un hiatus disparût, ou 
pour qu'une syllabe muette, après la quatrième syllabe accentuée, fût élidée, selon l'exigence moderne que rien ne justifie.

Aujourd'hui ce vers de six syllabes :

Le roi Marsile était à Saragosse,

Est juste ; et celui-ci :

Le roi Marsile revient à Saragosse,

Est faux parce que l'e final de Marsile n'est pas élidé. Cependant on ne compte dans aucun cas l'e final de Saragosse. Au moyen âge on n'eût pas compté davantage l'e final de Marsile, après la césure. Pourquoi ne pas faire de même en traduisant notre poëme ?

Petit de Julleville va chercher à perfectionner le système initié par d'Avril en supprimant certaines des règles qu'il s'était données et, surtout, en conservant l'assonance qui lui paraît un élément fondamental de la musique générée par la Chanson de Roland ${ }^{53}$ :

J'essaye donc de traduire le Roland, comme a fait M. d'Avril, vers pour vers, dans le rythme décasyllabique ; mais je conserve les règles de versification que le poëte a suivies. Je ne compte pas la syllabe brève qui suit la césure ; j'accepte l'hiatus et même l'inversion, pourvu qu'elle soit claire à l'esprit. Ces facilités m'ont permis d'être très-fidèle à l'original. J'aurais même pu traduire littéralement partout, si j'avais tenu à conserver les assonances, dont personne ne s'était préoccupé jusqu'ici. Tous les traducteurs avaient déclaré impossible de conserver l'assonance ; et M. d'Avril avait même dit, en la sacrifiant, " qu'elle parle peu aux yeux ». Elle est en tout cas un élément si essentiel dans la versification de notre poëme que tout d'abord je me suis attaché à la conserver le plus possible, et peu à peu à la conserver partout, afin que ma production sonnât, comme un écho fidèle du texte, à l'oreille de ceux qui ne lisant pas couramment le français $\mathrm{du} \mathrm{XI}^{\mathrm{e}}$ siècle, voudront néanmoins le connaître sous sa forme rajeunie mais non altérée.

Petit de Julleville précise encore que, pour parvenir à ses fins, « certains mots du texte n'exist[a]nt plus en français » et d'autres ayant vu leur «prononciation [...] modifiée », il a « dû plusieurs fois remplacer une assonance qui n'existe plus aujourd'hui par une autre correspondante, et cinq à six fois partager même une laisse en deux ou trois couplets, avec autant d'assonances différentes $»^{54}$. Concernant le lexique, il affirme enfin que : « tout en me faisant une loi générale de n'employer dans ma traduction que les termes de la langue actuelle, j'ai cru devoir conserver un certain nombre

53. Ibid.,p. 91-92.

54. Ibid., p. 92. 
de mots archaïques, indispensables, à ce qu'il m'a semblé, à l'exactitude et à l'énergie de ma traduction ${ }^{55}{ }$.

\section{De la musique avant toute chose}

Les traductions que nous avons vues jusqu'ici semblent s'efforcer toujours davantage de conserver le rythme du texte original. Si celle de Petit de Julleville paraît s'en être rapprochée aussi près que possible, d'autres traducteurs proposeront à leur tour des versions versifiées ou « rythmées » de la Chanson de Roland. Aucune traduction ne saurait contenir tout entière la voix du texte original. Il reste toujours quelque chose qui, lui ayant échappé, invite à la traduire à nouveau. C'est d'autant plus sensible ici que ce n'est pas simplement la Chanson de Roland que l'on veut traduire, mais la cantilena Rolandi qui résonne à travers elle, une chanson inconnue dont la présence tiendrait à la capacité de la traduction de susciter le même enthousiasme que celui qui poussa les soldats de Guillaume à se battre.

Dans sa traduction publiée en 1885 et destinée avant tout à l'éducation de la jeunesse, Édouard Rœhrich s'est souvent contenté de la prose. Mais il a « essayé en plusieurs endroits de traduire en vers certaines parties de la Chanson de Roland qui ne [lui] semblaient guère susceptibles d'être fidèlement reproduites en prose », une tâche qui, précise-t-il dans son introduction, «ne manquait pas d'être singulièrement délicate ${ }^{56}$. Il s'est efforcé pour cela « de reproduire purement et simplement en français les vers originaux, en conservant autant que possible leur rythme, leur syllabisme, et leur assonance ». Aussi s'est-il attaché à décrire de manière précise les principales règles de l'ancienne prosodie qu'il a respectées dans les passages traduits en vers, en particulier la structure du décasyllabe et l'assonance. S'il ne pense pas avoir réussi « à refaire les vers héroïques dans toute leur perfection », il espère « qu'on y sent malgré tout quelque chose du puissant souffle qui anime le poème $»^{57}$.

Cinq autres traductions en vers seront publiées avant la version de Bédier. En 1886, Amédée Jubert propose une traduction en laisses de diverses longueurs (sans qu'elles correspondent pour autant à celles du texte original), où alternent, liés par des rimes plates, des alexandrins et des décasyllabes ${ }^{58}$. Il ne s'en explique pas dans sa préface. Il se contente d'affirmer que, pour faire comprendre le poète de la Chanson de Roland, « une traduction vraiment heureuse de son chef-d'œuvre vaudrait mieux

55. Ibid., p. 103.

56. La Chanson de Roland, traduction nouvelle à l'usage des écoles précédée d'une introduction sur l'importance de la Chanson de Roland pour l'éducation de la jeunesse et suivie de notes explicatives, par É. RøenRICH, Paris, 1885, p. 117.

57. Ibid., p. 117 et 119.

58. La Chanson de Roland, traduite en vers par A. Jubert, Paris, 1886. 
que toute dissertation », et qu'il doit être possible de « reproduire dans notre langue le génie des ancêtres qui brille à chaque page de la Chanson ${ }^{59}$. Au lieu de s'attacher à rendre le rythme propre à cette dernière, Jubert semble plutôt l'utiliser comme un moyen de renouveler la prosodie française (rejoignant ainsi les expérimentations métriques et rythmiques des poètes symbolistes contemporains, de Rimbaud à Crise de vers de Mallarmé). C'est à quoi contribuerait la forme expérimentée dans sa traduction ${ }^{60}$ :

La poésie française qui, au dire de beaucoup, semblerait dépérir, n’a peutêtre besoin, pour revivre, que d'aller recevoir un nouveau baptême dans l'immense piscine du moyen âge.

Léon Clédat, qui avait publié en 1886 une nouvelle édition de la Chanson de Roland ${ }^{61}$, publiera un an après une traduction " archaïque et rythmée » à laquelle il consacre les dix pages de sa préface ${ }^{62}$. S'il reconnaît qu'on « est bien obligé de substituer aux mots, aux tournures et au rythme étrangers, les mots, les tournures et le rythme français qui s'en rapprochent le plus, [...] quand on veut traduire en français un ouvrage écrit dans une langue étrangère, langue morte ou vivante », il estime que, " quand il s'agit de faire comprendre au public de notre temps les chefs-d'œuvre de notre vieille littérature », il est « possible de procéder autrement et d'être plus fidèle ${ }^{63}$. Il en serait ainsi pour commencer du lexique ${ }^{64}$. Il en serait de même pour « la syntaxe dite de position » et pour « la syntaxe dite d'accord ${ }^{65}$. Il serait même possible de « conserver assez facilement le rythme de l'ancienne versification ${ }^{66}$ ». Par exemple, affirme Clédat, « "De sa main destre l' at asols et seigniét" devient "De sa main droite l'a absous et signé", qui est un vers de dix syllabes avec césure à la quatrième, comme celui du texte original » . $\mathrm{Si}$ « ce vers n'est pas conforme aux règles actuelles, puisqu'on y trouve un hiatus ( $a$ absous), et que l' $e$ de droite, venant après la césure, ne compte pas dans le vers quoiqu'il ne soit pas élidé, il suffit ou plutôt il importe » avant tout «que le vers soit conforme aux règles anciennes, puisque nous voulons donner une idée aussi complète que possible de l'ancien texte, et non point faire des vers modernes ». Lorsqu'un « mot français a aujourd'hui une syllabe de plus ou de moins que dans l'ancienne langue », comme il arrive souvent, Clédat s'est vu obligé de «modifier le texte » pour

59. Ibid., p. XXIII.

60. Ibid., p. XXIV

61. La Chanson de Roland, nouvelle édition classique, précédée d'une introduction et suivie d'un glossaire, par L. CLÉDAT, Paris, 1886.

62. La Chanson de Roland, traduction archaïque et rythmée, accompagnée de notes explicatives, par L. CLÉDAT, Paris, 1887.

63. Ibid., p. V.

64. Ibid., p. VI-IX.

65. Ibid., p. IX-X.

66. Ibid., p. XI-XIII. 
« conserver le rythme », mais il ne l'a fait, précise-t-il, qu'en « réduisant au minimum chaque modification, et en [se] conformant scrupuleusement aux anciens usages de la langue et de la versification ». Par exemple, il traduit «Guenes respunt » (au v. 529) par «Ganelon dit », plutôt que par «Ganelon répond », remplaçant le verbe répondre par le verbe dire pour compenser la syllabe supplémentaire introduite par la forme du cas régime Ganelon employée aujourd'hui à la place du cas sujet Guenes. Clédat a souvent été contraint, cependant, de « sacrifier l'assonance » («partie secondaire, en somme, de l'ancien rythme », affirme-t-il pour se justifier), afin de ne pas avoir à introduire de modifications plus importantes dans le texte. Mais il a choisi de conserver « cette partie de la cadence ancienne qui réside dans les terminaisons des vers uniformément masculines ou féminines pour une même laisse ». Lorsqu'il ne pouvait pas maintenir la fin du vers du texte original, le plus souvent « une légère modification de l'ordre des mots suffisait ». Par exemple, au lieu de « Mon parâtre est : ne veux que mot en sonnes » (v. 1027) dans une laisse en assonance masculine (l'ancien français donnant suns), il traduit par «Mon parâtre est : ne veux qu'en sonnes mot », estimant que, « dans la syntaxe du vieux français, l'ordre des mots [étant] fort libre, une modification de ce genre n'altère en rien le caractère de l'ancien vers ».

Clédat achève la présentation de sa traduction en reconnaissant que, « parmi les traductions déjà publiées de la Chanson de Roland, deux seulement ont été conçues d'après des idées voisines des [siennes] : ce sont les publications de M. d'Avril et de M. Petit de Julleville ${ }^{67}$. Sans chercher à «discuter [...] les méthodes suivies par ces deux traducteurs », il lui semble possible d'affirmer, "sans crainte d'être contredit », que sa « traduction se rapproche plus qu'aucune autre, et autant qu'il était possible, du texte primitif ». Grâce à elle, tous « ceux qui, sans avoir eu le loisir de se livrer à l'étude scientifique de l'histoire de la langue, s'intéressent assez à notre plus ancienne littérature », pourront « en pénétrer intimement l'esprit, le caractère, le style, et l'harmonie ». Le texte qui leur est offert, conclut Clédat, " conserve dans la plus large mesure les mots, les tournures, la syntaxe, le rythme de l'original, et il peut [...] donner une idée complète de la versification épique et de la langue même (sauf la forme des mots) du vieil auteur du Roland ». La traduction de Clédat veut donc à la fois rendre compréhensible le texte en ancien français et respecter le plus possible ses caractéristiques formelles afin qu'elles demeurent perceptibles.

«Il vaudrait mille fois mieux lire la Chanson de Roland avec intérêt, plaisir, émotion, le sens de quelques passages dût-il échapper, que de se renseigner exactement sur le moindre détail, tout en ne se laissant pas toucher et pénétrer par l'esprit du trouvère, si puissant dans sa naïveté »,

67. Ibid., p. XIII-XIV. 
affirme Maurice Bouchor dans l'avant-propos de sa traduction, destinée à « la jeunesse française », publiée en $1899^{68}$. Il aurait voulu employer un autre terme que celui de «traduction », car « on ne peut guère "traduire" un texte aussi éminemment français que celui de la Chanson de Roland; on essaie de le rajeunir ; mais, poursuit-il, chose tentée n'est chose accomplie, et le mot de traduction, quoique impropre, m'a semblé préférable à un autre, parce qu'il était moins ambitieux ${ }^{69} \gg$.

Bouchor affirme n'avoir fait « aucun usage » des nombreuses traductions en vers qui ont précédé la sienne, mais qui ont été composées selon des systèmes différents de celui qu'il s'est résolu de suivre : il ne les connaissait pas « avant de [s] e mettre au travail », assure-t-il, et il a cru «meilleur de ne pas [les] lire, pour ne recevoir d'impressions que du poème original ${ }^{70}$. Il s'est servi en revanche de la « traduction en prose » de Gautier. S'il n'a nullement la prétention de remplacer l'édition « classique » de Gautier (avec sa traduction, ses notes et son glossaire, qui a « sa place dans tous les établissements d'instruction où l'on fait des études littéraires approfondies »), il pense en revanche «qu'il peut être bon de mettre entre les mains de la jeunesse une Chanson de Roland dégagée de tout appareil d'érudition, et qui, au lieu de servir à de rares exercices de philologie, portant sur quelques pages du poème, soit lue couramment comme on lit une histoire faite pour intéresser, pour émouvoir, pour laisser au cœur une vive impression ${ }^{71}$. C'est pour cette raison qu'il a choisi de la traduire en vers.

Bouchor reconnaît tout d'abord qu'une « traduction en vers a le tort [...] de ne pouvoir être littérale ». Mais, soutient-il, « la stricte exactitude n'est guère plus facile à obtenir dans une traduction en prose. Il suffit de jeter les yeux sur la très estimable version de Léon Gautier pour voir qu'il a souvent, et à dessein, été inexact, sacrifiant la lettre pour sauver l'esprit, c'est-à-dire l'intention, la nuance, l'énergie du texte ». C'est ce que Bouchor entend faire lui aussi, afin d'offrir une traduction qui détienne le même pouvoir d'émotion que le texte original, quitte à ne pas être littérale, plutôt que d'être fidèle à sa lettre et en perdre les qualités poétiques. S'il a « toujours recherché à mettre en vive clarté les traits essentiels et caractéristiques de ce poème », il n'a pas renoncé pour autant « à cette liberté qui est, pour toute œuvre d'art, la première condition de la vie $»^{72}$. D’ailleurs, précise-t-il :

68. La Chanson de Roland, traduite en vers par M. Bouchor, à l'usage des Écoles normales, lycées et collèges, écoles primaires supérieures, cours complémentaires, Paris, 1899 , p. 2 et 3.

69. Ibid.

70. Ibid., p. 7.

71. Ibid.

72. Ibid., p. 7-8. 
Un poème est $[\ldots]$ chose bien incomplète lorsque, dans une version même très exacte, il perd le rythme, l'accent, la musique du vers. Cela est vrai surtout d'une épopée, faite pour être dite bien plutôt que pour être lue, car l'immense majorité des auditeurs primitifs de la Chanson de Roland ne savaient pas lire. Malgré la différence des temps, le caractère du poème exige toujours qu'il soit récité à haute voix et, s'il est possible, devant un assez nombreux auditoire.

Une traduction en prose, apparemment plus exacte, risquerait donc d'être, en réalité, bien plus infidèle qu'une traduction en vers, car elle ne se prêterait guère à une déclamation soutenue ${ }^{73}$.

L'emploi du vers n'est pas la seule raison de la liberté que Bouchor s'est donnée à l'égard du texte original. Comme Vitet et Jônain avant lui, il s'est également permis de le raccourcir. D'une part, il a «ça et là resserré le texte, qui parfois - estime-t-il - s'affaiblit en se répétant ». D'autre part, il a supprimé l'épisode de Baligan, qui lui paraît - comme c'était également le cas pour certains philologues - « être une addition fâcheuse au poème primitif ». Mais, assure Bouchor ${ }^{74}$ :

Malgré les libertés que j'ai prises, mon seul dessein a été de communiquer à d'autres la profonde impression que me fait à moi-même la Chanson de Roland. Ma traduction est un essai pour rendre cette impression dans toute sa sincérité comme dans toute sa force. Si, en traduisant le poème, j’y ai ajouté du mien, ce fut d'une façon tout involontaire ; et je crois bien avoir été toujours fidèle à l'esprit du texte, même dans mes inexactitudes matérielles.

On comprend que Bouchor ait pu hésiter à qualifier son travail de traduction. Traduire ici est moins rendre le sens véhiculé par la lettre du texte, que l'impression que celui-ci a pu exercer sur son lecteur. C'est la restitution de cette impression qui caractériserait la véritable fidélité du traducteur, quitte à ce que ce dernier prenne quelques libertés avec le texte original et y ajoute ce que celui-ci a évoqué dans son esprit mais dont on ne saurait pour autant lui refuser totalement la propriété. Ce terme, que Bouchor répète à plusieurs reprises et qui prendra une importance particulière chez Proust, rapproche du même coup sa traduction de l'esthétique mise en valeur par la peinture impressionniste.

La présentation du vers qui lui servira à traduire la Chanson de Roland est rejetée par Bouchor à la fin de son ouvrage dans une note « Sur la versification du poème original et de la présente traduction ${ }^{75}{ }$. Il commence par rappeler les trois traductions en vers de d'Avril, de Lehugeur et de Petit de Julleville, qu'il n'aurait lues qu'après avoir réalisé la sienne. Il reproche

73. Ibid., p. 8. Bouchor a d'ailleurs ajouté au terme de son ouvrage une note intitulée « Indication relative aux passages à lire en public », p. 163-167.

74. Ibid., p. 8-9.

75. Ibid., p. 159-162. 
tout d'abord à Lehugeur d'avoir substitué au décasyllabe, qui certes est « un peu maigre, un peu sec, mais très net, très rapide, très français », « notre grand vers de douze syllabes, dont la majestueuse ampleur n'est point ici à sa place, et qui doit forcément délayer le texte du poème, en même temps qu'il en altère la physionomie ». Il reproche ensuite à Petit de Julleville d'avoir conservé l'assonance, qu'il considère comme « une sorte de rime incomplète ». Certes, reconnaît-il, « nos vieux poèmes français étant écrits en vers assonancés, il est naturel que l'idée de revenir à l'assonance soit venue à un traducteur de la Chanson de Roland. Mais - poursuit-il - les oreilles modernes, habituées à la rime, trouvent l'assonance bien pauvre ; et il m'a semblé que le retour à une forme primitive de notre versification aurait quelque chose d'artificiel ». C'est à plus forte raison, enfin, qu'il reproche à d'Avril d'avoir « adopté le système d'écrire en vers blancs, c'està-dire sans rimes ni assonances ${ }^{76}$.

Bouchor comprend très bien que le choix du vers blanc ou assonancé a permis, « en écartant les difficultés de la rime, [de] traduire vers par vers le poème original et en donner une version aussi exacte que possible ». Mais, poursuit-il en revenant sur sa conception de la traduction qu'il avait présentée en introduction, « l'exactitude » lui « semble très différente de la fidélité vraie ». On ne saurait donc toujours la conserver ${ }^{77}$ :

Comme la Chanson de Roland a été, en son temps, une œuvre jeune, libre et vivante, l'essentiel était, je crois, d'en bien pénétrer et d'en bien rendre l'esprit, avec vie et liberté ; et pour cela, pour donner l'impression de ce que fut le poème en sa nouveauté, la rime ne pouvait être une entrave à quiconque a l'instinct et la pratique du vers français, tel que la tradition nous l'a façonné.

J'adoptai donc le vers de dix syllabes rimés.

Si le décasyllabe conserve le vers du texte original, l'emploi de la rime permettrait de rendre compte de l'esprit d'invention et de nouveauté qu'a dû porter la Chanson de Roland au moment de sa création.

Reste la question des laisses. La rime ayant remplacé l'assonance, on ne saurait s'en tenir à une seule et même rime tout au long d'une laisse. Bouchor s'étant résigné « à faire sagement rimer les vers deux par deux », il lui fallait « une suite de strophes où l'on retrouvât à peu près le mouvement des laisses, dont chacune marque un moment du récit, et que la rime y eût assez d'éclat pour compenser, en peu de vers, l'effet d'assonances nombreuses ». C'est pourquoi il se décida « pour une strophe de huit vers, construite sur trois rimes différentes », se donnant pour modèle le huitain

76. Ibid.,p. 159-160.

77. Ibid., p. 160-161. 
des Testaments de Villon ${ }^{78}$. Une même laisse peut alors se partager en deux strophes ou se « résumer en un seul » couplet. Bouchor précise qu'il s'est « conformé à l'alternance des rimes masculines et des rimes féminines, qui a prévalu dans notre versification depuis le $\mathrm{XVI}^{\mathrm{e}}$ siècle », et que, « tout en maintenant la division du vers original en deux parties, la première de quatre syllabes, la seconde de six », il a « cherché à varier les effets en mettant parfois le repos après d'autres syllabes que la quatrième ». Aussi, affirme-t-il, « il faut y faire attention pour bien lire le poème à haute voix », la «ponctuation, ou, d'une façon générale, le sens de la phrase, indiqu[ant] le repos ». Il termine enfin sa note sur la versification de la Chanson de Roland par une série de recommandations concernant la lecture à voix haute de sa traduction ${ }^{79}$ :

Il faut aussi, comme dans toute lecture d'un poème, accentuer davantage les mots essentiels, ceux qui contiennent le plus d'émotion, de pensée, de couleur, de sonorité.

On ne doit pas toujours marquer un arrêt à la fin du vers. Les rejets d'un vers à l'autre sont assez fréquents ; surtout les rejets de quatre syllabes.

Quelquefois, c'est d'une strophe à l'autre qu'il faut passer très rapidement. Il convient de lire sur un ton de voix soutenu, avec chaleur ou émotion, mais toujours avec naturel et d'une façon assez vive, sauf lorsqu'on veut exprimer certains sentiments graves et tendres, marquer l'hésitation ou indiquer le passage d'un ordre d'idées à un autre. En ce dernier cas, il ne faut pas craindre de prendre du temps. On donnera beaucoup de relief aux dialogues, de façon à évoquer pour l'auditeur la physionomie et le geste des personnages, je dirais presque leur son de voix, si je ne craignais que ce conseil fût interprété d'une façon trop matérielle.

Si c'est aux élèves des écoles que Bouchor destinait sa publication, c'est à « l'Armée nationale » que Joseph Fabre dédie en 1902 la traduction qu'il offre à son tour de cette «épopée du patriotisme ${ }^{80}$ ». Comparé à l'Iliade, ce «bréviaire du soldat français » est destiné à « rallumer le foyer où s'alimenta la flamme guerrière des croisés » afin de venger la défaite de $1870^{81}$. Aussi Fabre ne manque-t-il pas de rappeler à nouveau que « de vieux textes montrent, à la bataille d'Hastings, le jongleur Taillefer et les soldats normands entonnant la chanson des vaincus de Roncevaux pour s'animer à la victoire, et transformant la complainte funèbre en hymne

78. Ibid., p. 161.

79. Ibid., p. 162.

80. J. Fabre, La Chanson de Roland. Traduction nouvelle et complète, rythmée conformément au texte roman, précédée de Roland et la Belle Aude, prologue à La Chanson de Roland, et suivie de Autour de Roland, échos des chansons de geste de la vieille France, Paris, 1902, p. 4.

81. Ibid., p. 11. 
de triomphe ${ }^{82} »$. " C'est une poésie frustre, primitive, d'allure raide et d'haleine courte ${ }^{83} »$, qui doit «nous délivrer [...] de cette écriture artiste, foncièrement artificielle » à laquelle semble se complaire selon lui la littérature contemporaine ${ }^{84}$.

La traduction proposée par Fabre entend restituer aussi bien « le sens » que «la poésie du vieux chef-d'œuvre », soit à la fois « la clarté, la netteté, l'aisance sans lesquelles on n'a pas de lecteurs », et « le mouvement, le coloris, le pathétique nécessaires pour ne pas trahir le modèle ». En ce qui concerne ce dernier aspect, Fabre a choisi de suivre « d'un bout à l'autre le rythme du texte original ». En effet, soutient-il, « ne pas avoir le constant souci de la cadence du vieux texte, c'est se résigner à ne pas en rendre la couleur et la poésie ${ }^{85}$. Mais qu'en est-il de ce "rythme » ou de cette «cadence»? Ils ne se réduisent ni à l'assonance ni à la rime. Fabre refuse de traduire en rimes, "parce que les nécessités de la rime entraineraient inexactitudes et remplissage » et « qu' une traduction en rime [...] constituerait une altération sérieuse du texte original ». Mais il renonce également à conserver l'assonance, pour ne pas avoir « des couplets de dix ou vingt vers monorimes » qui provoqueraient non seulement une « inévitable monotonie », mais aussi d'inévitables «infidélités ${ }^{86}$. L'assonance et la rime ne sauraient donc se confondre avec le « rythme » qui caractérise la poésie. Si « la rime est l'ornement du vers », est-il précisé en note, « c'est le rythme qui en est l'essence ${ }^{87}$. Mais Fabre n'en dit guère plus. En guise d'illustration - ou d'argument - et pour permettre au lecteur de se faire son avis sur la question, il cite un extrait de trois traductions différentes parmi celles qui l'ont précédé, à la suite de l'extrait correspondant de sa propre « traduction rythmée » : soit « un extrait de la traduction en prose libre de Léon Gautier », «un extrait de la traduction en vers de M. Maurice Bouchor», et un «extrait de la traduction assonancée de Petit de Julleville ${ }^{88}$. Pour savoir ce qui caractérise la « traduction rythmée » de Fabre, il ne nous reste donc qu'à la comparer avec ces traductions et en analyser le « rythme » particulier. On peut noter tout d'abord qu'elle est en décasyllabes et que la structure métrique recouvre régulièrement la structure syntaxique. Mais le «rythme » ne porte pas seulement sur l'unité du vers. Fabre distingue aussi « divers développements, formant chacun un tout dont les parties se répondent », chaque développement pouvant constituer « une véritable symphonie poétique, se déroulant avec une progression harmonieuse où

\footnotetext{
82. Ibid., p. 12.

83. Ibid.,p. 18.

84. Ibid.,p. 16.

85. Ibid., p. 22-23.

86. Ibid., p. 23-24.

87. Ibid., p. 25 , n. 1

88. Ibid., p. 25-26.
} 
domine un thème principal qui joue le rôle du refrain ou du leit motive ${ }^{89}$. Il rappelle du même coup que « la Chanson de Roland fut faite non pour être lue, mais pour être entendue » :

Aussi est-ce surtout quand elle est dite devant un public, que deviennent sensibles les harmonies que j'y signale. Même des enfants, des illettrés, sans savoir le pourquoi, s'en montreront touchés. Dès lors, je ne souhaiterais rien tant à la présente traduction que la faveur de quelques lectures publiques faites dans les écoles ou dans les salles de conférences, en donnant un juste relief au rythme des couplets et à ces expressions reprises du récit, à ces retours gradués des motifs dominants, d'où résulte un crescendo d'intérêt et d'émotion ${ }^{90}$.

Pour autant qu'il soit parvenu à restituer le « rythme » du texte original, Fabre espère que sa traduction sera capable à son tour de « faire sentir l'ivresse et le frisson des combats » que suscitait cette chanson ${ }^{91}$.

C'est à ses « élèves de Fontenay-aux-Roses » qu'Henri Chamard dédie en 1918 sa traduction (publiée l'année d'après), non seulement parce qu'elles ont pu suivre les conférences qu'il a données sur le sujet, mais aussi parce que « depuis quatre ans » elles vivent « chaque jour une vie d'héroïsme et d'abnégation » et qu'elles voient « engagés dans les hasards et les périls de la plus terrible des guerres tant de héros chers à [leurs] cœurs, [leurs] pères, [leurs] frères », et pour certaines leurs « fiancés ${ }^{92}$. Destinées à « former des âmes », c'est-à-dire à « instruire et façonner la studieuse jeunesse de ce peuple de France, si courageux, si généreux, qui pour la plus sainte des causes, a versé sans compter le meilleur de son sang, et d'où nous avons vu sortir tant de Rolands et d'Oliviers », elles sont invitées à lire à leurs futurs élèves « quelques pages du vieux poème », et à leur montrer ainsi « le lien toujours vivace qui joint au passé le présent ». Soit ${ }^{93}$ :

Ce pieux attachement à certaines idées que, sur notre sol bien aimé, se transmettent les générations - ardent amour de la patrie, culte souverain de l'honneur et de la parole donnée, crainte de forfaire et d'être honni, besoin de se dévouer pour la justice et le droit, tendresse et pitié pour les faibles, croyance à l'idéal -, tout cet ensemble de vertus que nos aïeux du Moyen Age, pénétrés de foi religieuse, nommaient du nom de chrétienté, que nous résumons aujourd'hui du nom de civilisation, et qui sont le trésor le plus pur de la race!

89. Ibid., p. 39.

90. Ibid., p. 39-40.

91. Ibid., p. 12.

92. La Chanson de Roland. Traduction nouvelle d'après le manuscrit d'Oxford, par H. Chamard, Paris, 1919, p. III.

93. Ibid., p. IV. 
Constatant dans sa préface qu'on «a traduit déjà bien des fois en français la Chanson de Roland ( « en vers, soit rimés, soit assonancés », « en prose libre, en prose rythmée, en prose mêlée de vers »), Chamard n'a pas craint, dit-il, «d'en profiter $»^{94}$. Il estime en même temps que sa « tentative » possède une « vraie nouveauté » ${ }^{95}$. D'une part, contrairement à certains de ses prédécesseurs, il a « suivi très fidèlement le manuscrit d'Oxford » dont Bédier avait souligné la valeur. D'autre part, « convaincu $[\ldots]$ que l'on ne traduit bien les poètes qu'en vers, et que la prose, même rythmée, ne suffit pas à leur garder le mouvement et la couleur » », il a réalisé une « traduction en vers ». Il ne croit pas, en effet, « que nos oreilles, habituées à la rime depuis tant de siècles, puissent se contenter de la simple assonance », d'autant qu'il semble inutile de vouloir refaire ce qu'a tenté Petit de Julleville. Chamard espère toutefois que les principes qu'il a suivis permettront de rapprocher sa traduction « de l'original d'une manière assez sensible ${ }^{96}$. Ces principes ne sont pas les mêmes que ceux des autres traductions rimées. Chamard estime en particulier que « rendre la Chanson de Roland en alexandrins à rimes plates, comme Alfred Lehugeur, ou, comme Maurice Bouchor, dans la forme artistique du huitain villonesque, c'est se tromper semblablement sur la facture du vieux trouvère, qui n'a manié que le décasyllabe et qui les a groupés en laisses inégales ${ }^{97}{ }^{\gg}$.

Chamard conserve donc le décasyllabe et traduit " laisse par laisse, vers par vers ». Ne pouvant réaliser des laisses monorimes, il s'est rallié au «système des rimes libres », qui lui procure - estime-t-il - «le double avantage d'utiliser beaucoup de rimes fournies par le modèle, et de multiplier les rimes similaires, ce qui maintient, dans une certaine mesure, l'unité de la laisse $»^{98}$.

Deux considérations ont guidé plus précisément Chamard dans son travail. D'une part, « les vers du Roland primitif ét[ant] destinés, non à la lecture, mais à l'audition », il a tenté « une version moderne où l'oreille serait le seul juge du rythme et de la rime ». Aussi, « puisque notre oreille, aujourd'hui, ne perçoit plus de différence, pour la quantité des syllabes, entre armée et armés [etc.] », il n'a pas tenu compte, dans la mesure du vers, du $e$ muet. En outre, « puisque notre oreille, aujourd'hui, ne perçoit plus de différence, au point de vue du son final, entre manoir et noire [etc.] », il ne distingue pas, à la rime, les mots masculins des mots féminins, ni les mots singuliers des mots pluriels ${ }^{99}$. D'autre part, « dès lors que le rythme et la rime satisfont pleinement l'oreille », il a «négligé l'alternance systématique des

\footnotetext{
94. Ibid.,p. V.

95. Ibid., p. V-VI.

96. Ibid.,p. VI.

97. Ibid., p. VI-VII.

98. Ibid., p. VII.

99. Ibid., p. VI-VIII.
} 
masculines et des féminines, et l'obligation établie depuis la Renaissance de faire rimer richement certaines catégories de mots », rejetant du même coup « la règle qui veut qu'on rime autant pour l'œil que pour l'oreille », se permettant quelquefois, « comme au Moyen Age, la rime du simple et du composé », admettant même l'hiatus « quand l'hiatus était [...] le seul moyen de rester fidèle à l'original ». Chamard constate du même coup que, « sans l'avoir cherché » (mais comme Jubert avant lui), il s'est «trouvé pratiquer en fait, pour rendre un vieux texte neuf fois séculaire, certaines libertés de notre poésie contemporaine », et que « c'est grâce à ces libertés [qu'il a] pu parvenir à plus d'exactitude, et qu'un grand nombre de [ses] vers ne sont, en somme, qu'une transcription pure et simple des vers de l'œuvre primitive ${ }^{100}$.

Chamard a cherché aussi à « garder au poème sa couleur archaïque ». Pour cela, il a fait « appel, par certains mots, par certains tours, aux ressources du vieux langage ${ }^{101}$. D'une part, sur le plan lexical, il a conservé « les termes techniques de vêtement et d'armure » (comme bliaud), calqué parfois « sur l'original des mots intraduisibles» (comme aumaçour), maintenu de « vieux mots » dont «le sens n'est pas obscur» (comme chef pour tête) ${ }^{102}$. D'autre part, sur le plan syntaxique, il a eu recours « à certaines souplesses de syntaxe et de style qui n'étaient pas encore toutes passées d'usage au début du XVII ${ }^{\mathrm{e}}$ siècle », s'autorisant parfois à supprimer « l'article indéfini ou défini », à pratiquer "l'inversion du complément direct », à faire «l'ellipse du pronom personnel sujet ${ }^{103}$. De même que la versification proposée par Chamard s'efforce d'allier la prosodie de l'œuvre originale et l'oreille des auditeurs contemporains, la coloration archaïsante de sa langue semble vouloir joindre le passé au présent.

\section{La fin du chant}

Trois traductions en prose ont été publiées durant les cinquante ans qui séparent la version de Gautier, parue en 1872, et celle de Bédier, publiée en $1922^{104}$. Les traductions rythmées ou versifiées furent en revanche nettement plus nombreuses. Le travail de Bédier inversera toutefois la tendance de manière significative et durable.

Avant de publier son édition et sa traduction de la Chanson de Roland, Bédier avait fait paraître une monumentale étude consacrée à la

100. Ibid., p. VIII.

101. Ibid.

102. Ibid., p. IX.

103. Ibid., p. IX-X.

104. La Chanson de Roland, traduction nouvelle, revue et annoté par H. Feullereret, Limoges, 1879 ; La Chanson de Roland, trad. A. Снаillot, Limoges, 1880 ; La Chanson de Roland. A Modern French Translation, par G. GedDes, New York, 1906. 
chanson de geste, dont une partie importante est consacrée à la Chanson de Roland ${ }^{105}$, ainsi que deux études portant principalement sur la précellence du manuscrit d'Oxford, les qualités littéraires de son texte et la méthode qu'il entend suivre pour en assurer l'édition ${ }^{106}$.

Bédier ne destine pas son ouvrage « aux seuls érudits ». Il estime en effet «que tous les lettrés » doivent pouvoir « lire le poème vénérable et s'y plaire ». C'est « à leur intention, pour leur rendre l'effort moins rude et les assister chemin faisant », qu'il « imprime en regard du texte ancien, une transcription de ce texte en langage d'aujourd'hui, une « traduction » », ainsi que l'avaient fait avant lui, précise-t-il en ne retenant que ces deux noms, Génin et Gautier ${ }^{107}$.

C'est donc aux « lettrés » que s'adresse Bédier, non à la jeunesse et aux élèves des écoles, aux soldats de l'Armée nationale ou au peuple français tout entier. Contrairement à la plupart de ses prédécesseurs, son ouvrage n'est pas destiné à être chanté ou déclamé, mais à être lu. Sa traduction est chargée principalement d'assister le lecteur afin qu'il puisse suivre plus facilement le texte original. Elle n'entend nullement se substituer à ce dernier et n'a donc pas à s'efforcer d'en transposer les caractéristiques formelles. Aussi Bédier ne prétend-il «qu'à l'exactitude littérale », se contentant d'une traduction qui ne serait qu'une simple «transcription », d'autant que, comme nous l'avons déjà vu, il soutient avec Du Bellay (mais contre Chamard et plusieurs des traducteurs de cette chanson avant lui), qu'il n'est pas possible de traduire les poètes ${ }^{108}$. Le seul moyen d'accéder $\mathrm{au}$ « rythme » et au «nombre » de cette chanson, serait de la lire dans la langue originale.

Ce constat négatif n'est toutefois pas la seule - ni peut-être la véritable - raison qui a entraîné Bédier à préférer une traduction en prose. En effet, affirme-t-il ${ }^{109}$ :

Il est un caractère de la Chanson de Roland que je crois avoir reconnu et senti avec une vivacité assez particulière et que, dans ma traduction, je me suis attaché, de toute ma ferveur, à sauvegarder. C'est bien à tort, il me semble, que

105. J. BÉDIER, Les Légendes épiques. Recherches sur la formation des chansons de geste, Paris, 1908-1913 (4 vol.), t. III, p. 183-477.

106. ID., « De l'autorité du manuscrit d'Oxford pour l'établissement du texte de la Chanson de Roland », Romania, 41 (1912), p. 331-345 ; ID., « L'art et le métier dans la Chanson de Roland», Revue des Deux Mondes, 13 (1913), p. 292-321. Sur les travaux que Bédier a consacrés à cette œuvre, $c f$. C. LuCKEN, « Joseph Bédier et la Chanson de Roland», Romanische Studien, 7 (2017), p. 97-117 ; et plus généralement, A. Corbellari, Joseph Bédier. Écrivain et philologue, Genève, 1997.

107. La Chanson de Roland, trad. J. BÉDIER, p. XII-XIV. Sur cette traduction, voir aussi A. Corbellari, « Traduire ou ne pas traduire : le dilemme de Bédier. À propos de la traduction de la Chanson de Roland», Vox romanica, 56 (1997), p. 63-82.

108. La Chanson de Roland, trad. J. BéDIER, p. XIV.

109. Ibid., p. XV-XVI. 
tant de critiques ont déploré la pauvreté des moyens d'expression du poète, ont cru devoir chercher des excuses à ce qu'ils appellent sa «gaucherie », sa «naïveté toute populaire ». J'admire au contraire les allures aristocratiques de son art, les ressources et la fière tenue, très raffinée, d'une langue ingénieuse, nuancée, volontaire, et qui révèle un souci constant de distinguer l'usage vulgaire du bon usage. Ce style est déjà d'un classique, il est un style noble. Dès le début du XII ${ }^{\mathrm{e}}$ siècle, la France des premières croisades tend de la sorte à créer, à constituer en dignité, par-dessus la diversité et la rusticité de ses dialectes et de ses patois, cette merveille, une langue littéraire.

Au lieu de la «gaucherie » ou de la « naïveté »d'une chanson barbare telle que pouvait l'imaginer la tradition romantique, Bédier y voit un style noble et aristocratique, un art raffiné, une « langue littéraire ». À l'épopée primitive que semblait impliquer la cantilena Rolandi entonnée lors de la bataille de Hastings succède une œuvre classique. Plutôt que de réciter au peuple illettré une poésie guerrière susceptible de l'émouvoir, Bédier offre aux « lettrés » une œuvre qu'ils pourront lire. Alors que le vers devait paraître lié à l'oralité d'un chant de guerre, la prose a dû lui sembler comme le meilleur moyen de rendre les caractéristiques qu'il reconnaît à la Chanson de Roland.

Comment Bédier s'y est-il pris plus précisément pour parvenir à cette « qualité souveraine du vieux maître » qu'est la « noblesse » ${ }^{110}$ ? Il affirme n'avoir cherché à restaurer ni sa syntaxe ni son lexique. Mais s’il refuse d'archaïser, il a entrepris de « répondre à l'effort du poète par un effort qui ressemblât au sien », en évitant « les mots récents, comme il évitait les mots bas » (ce qui me paraît correspondre davantage, en fait, aux normes de la tradition classique qu'à celles de la Chanson de Roland ou de la littérature médiévale). À l'exception des « termes techniques qui désignent des choses d'autrefois », il s'est efforcé « de n'employer que des mots et des tours bien vivants encore, mais qui, persistant tous dans notre usage, pussent tous se prévaloir de très anciens titres, plus anciens que la Renaissance ${ }^{111}$. C'est à ce titre, celui qui fait la noblesse d'un lexique ni trop archaïque ni trop récent, que la traduction de la Chanson de Roland peut espérer incarner le classicisme intemporel du français. Ce n'est donc pas à son chant que cette œuvre doit son prestige, mais à son lexique (à quoi tient aussi en grande partie la couleur qu'entendait privilégier Gautier). Seul le lexique paraît intéresser ici le traducteur en quête d'une langue qui, conforme à une tradition remontant en particulier à Malherbe et aux Remarques sur la langue française de Vaugelas (1647), se réduirait désormais au « bon usage ». Le vers n'est d'aucune utilité pour cela, bien au contraire. La prose suffit largement. 
Une autre motivation me semble toutefois pouvoir expliquer aussi bien la volonté qu'a Bédier de rattacher la Chanson de Roland à la tradition littéraire classique que sa traduction en prose. Pourtant, il n'en dit rien. Mais son silence me semble particulièrement éloquent. Depuis 1870 en tout cas, la plupart des critiques et des traducteurs français de la Chanson de Roland justifient les travaux qu'ils consacrent à cette œuvre par sa valeur morale et pédagogique, soulignant principalement le rôle qu'elle est susceptible de jouer pour renforcer l'identité nationale et, en particulier, pour encourager les Français à lutter contre l'envahisseur prussien ${ }^{112}$. En 1861 déjà, Jônain concluait sa préface en s'écriant «A moi Roland et Olivier, la vaillance, l'amitié, la patrie ! En avant pour la douce France ${ }^{113}$ ! » Lehugeur achève sa propre préface, datée de juin 1870, en affirmant qu'il a eu pour but « de faire que chacun pût lire [...] un livre où respirent, du commencement à la fin, la magnanimité chevaleresque, le culte de la patrie et de l'honneur, sous les traits et sous les noms de la France et de ses fils ${ }^{114}{ } »$. Le 8 décembre 1870, alors que Paris est encerclé, Gaston Paris consacre sa leçon d'ouverture au Collège de France à « La Chanson de Roland et la nationalité française ${ }^{115}$ » . Notant au début de son introduction qu'il écrit « au milieu des malheurs de la Patrie », Gautier souhaite en 1872 que « les beaux vers de ce Chant national [puissent] consoler ceux qui pleurent aujourd'hui sur leur pays » et que « ce récit du passé [puisse] nous rendre la confiance en l'avenir ! » ${ }^{116}$. Rœhrich consacre en 1885 l'essentiel de sa longue introduction à démontrer l'importance de cette chanson " pour l'éducation de la jeunesse », estimant qu'elle « est la plus belle glorification du patriotisme, du courage hérö̈que, de l'amitié, et du sentiment moral et religieux $\gg{ }^{117}$. Jubert demande en 1886 que les Français se reconnaissent « tout entiers dans la Chanson de Roland, expression et pensée : car elle est le miroir où s'est le mieux reflétée notre image $^{118}$ ». La même année, Clédat justifie « l'introduction de la Chanson

112. $C f$. B. Cerquiglini, "Roland à Roncevaux ou la trahison des clercs », Littérature, 42 (1981), p. 40-56 ; J. J. DugGan, « Franco-German Conflict and the History of French Scholarship on the Song of Roland », dans O. J. Gallacher et H. Damico éd., Hermeneutics and Medieval Culture, Albany, 1989, p. 97-106 ; C. Ridoux, Évolution des études médiévales en France de 1860 à 1914, Paris, 2001, p. 157-162, 613-624 ; M.-M. CASTELlanI, « Roland, héros de la Patrie française dans les préfaces aux traductions de la Chanson de Roland (18701919) ", dans C. Cazenave et F. Marchal-Ninosque éd., Mourir pour des idées, Besançon, 2008, p. 189-205 ; T. VAN HeMELRYCK, « La Chanson de Roland aux $\mathrm{XIX}^{\mathrm{e}}, \mathrm{XX}^{\mathrm{e}}$ et XXI siècles. De la glorification nationale à l'instrumentalisation idéologique », Interférences littéraires, 3 (2009), p. 27-35 ; et mon étude déjà citée, «Actualité de la Chanson de Roland... ».

113. La Chanson de Roland, trad. P. JônaIN, p. XIV.

114. La Chanson de Roland, trad. A. Lehugeur, p. XVI.

115. G. PARIs, « La Chanson de Roland et la nationalité française », dans La Poésie du Moyen Âge, Paris, 1885 , p. 87-118.

116. La Chanson de Roland, éd. et trad. L. GauTIER, p. VIII.

117. La Chanson de Roland, trad. E. Rerrich, p. 1 et 6-7.

118. La Chanson de Roland, trad. A. JUBERT, p. XIV. 
de Roland dans l'enseignement secondaire [...] non seulement par le grand mérite littéraire de ce vieux poème, mais encore par sa haute valeur morale ${ }^{119} \gg$ :

Du premier au dernier vers, il respire un ardent amour de « douce France », un profond sentiment de l'honneur et du devoir. Nous ne pouvons nous dissimuler qu'aujourd'hui plus que jamais il importe de former les jeunes générations au culte de la patrie : en lisant la Chanson de Roland, en admirant les sentiments qui animent Roland et ses glorieux amis, elles apprendront à sentir comme eux, à servir leur pays sans défaillance, à « mieux aimer mourir que laisser honnir la France».

Petit de Julleville soutient en 1894 que « la Chanson de Roland [...] est le plus ancien monument de notre nationalité » et que « ce n'est pas seulement la poésie française qu'on voit naître avec ce poème. C'est la France elle-même ${ }^{120}$. Offrant « le fruit de son travail à ces jeunes hommes, à ces jeunes filles, qui sont aujourd'hui l'espérance de la Patrie et qui, demain, seront sa force », Bouchor affirme en 1899 s'être efforcé de « mettre en lumière la haute valeur éducatrice de notre vieille épopée nationale ${ }^{121}$. Dédiant cette «épopée du patriotisme » à « l'Armée nationale », Fabre ne craint pas de rallumer en 1902 « le foyer où s'alimenta la flamme guerrière des croisés » tant qu'il y aura des « revanches » à prendre ${ }^{122}$. Enfin, en 1918, Chamard dédie à ses élèves la traduction qu'il a lue avec elles durant la guerre afin que « cette œuvre si naïve, mais d'un souffle si mâle, réconforte [leurs] sentiments et soutienne [leur] énergie », et qu'elles y puisent « tout le suc des vertus ancestrales ${ }^{123}$.

On ne trouve rien d'analogue dans l'avant-propos de Bédier. Il ne parle pas d'une éventuelle valeur morale et nationale de la Chanson de Roland. Il ne dit pas non plus qu'elle a été entonnée par un certain Taillefer lors la bataille d'Hastings, comme le font la plupart de ses prédécesseurs ${ }^{124}$. Bien qu'il conserve le titre qui lui est donné depuis Francisque Michel, il ne dit pas que cette chanson était le plus souvent chantée, qu'il faudrait aujourd'hui encore la réciter ou la lire à haute voix, et qu'elle s'adresse donc avant tout à l'oreille d'un auditeur plus qu'aux yeux d'un lecteur. Au contraire, il en parle comme un « livre » dont il célèbre la « langue littéraire » et qui, adressé aux « lettrés », semble destiné principalement à être $l u$ comme tout texte classique. Ce n'est pas que Bédier soit opposé

119. La Chanson de Roland, éd. L. CLÉDAT, p. XII.

120. La Chanson de Roland. Histoire, analyse, extraits, avec notes et glossaire, par L. Petit de Julleville, Paris, 1894, p. 22.

121. La Chanson de Roland, trad. M. Bouchor, p. 3 et 19.

122. J. FABRE, La Chanson de Roland, p. 5 et 11.

123. La Chanson de Roland, trad. H. CHAMARD, p. IV.

124. Bédier ne mentionne pas non plus la cantilena Rolandi dans ses Légendes épiques, dont il semble ainsi contester l'importance en même temps qu'il rejette la théorie des cantilènes. 
à l'usage qu'on peut en faire pour « fortifier [...] le sentiment national » des Français ${ }^{125}$. Mais on peut penser que la guerre qui vient de s'achever - avec les terribles souffrances qu'elle avait causées - ne permet plus à ce « sentiment national » d'être porté par un chant guerrier poussant le peuple français à se battre contre l'ennemi, mais qu'il est bien mieux représenté désormais par une œuvre dont la langue et le style empreints de «noblesse » lui permettent, non pas de ranimer la passion des combats, mais de contribuer au prestige littéraire et culturel de la France.

Les traductions rythmées ou versifiées qui précèdent la version de Bédier insistent tout particulièrement sur la capacité de la Chanson de Roland à insuffler l'amour de la patrie et à transmettre un certain nombre de valeurs morales. Elles l'inscrivent du même coup dans le prolongement de la cantilena Rolandi chantée à la bataille de Hastings (même si elles peuvent hésiter à les identifier). Le chant semble avoir une force particulière qui lui permet d'agir sur ses auditeurs en touchant directement leurs émotions plutôt qu'en sollicitant leurs réflexions, leurs connaissances ou leur goût. " N'est-il pas évident qu'à la guerre, les âmes des combattants sont enflammées par le son des trompettes [tubarum carmine]?» affirmait par exemple Boèce dans son De musica ${ }^{126}$. Aussi les auteurs de ces traductions s'intéressent-ils tout particulièrement à la prosodie de la Chanson de Roland, lui consacrant souvent dans leur préface une analyse détaillée qui en précise les caractéristiques avant d'expliquer comment elles pourront être transposées en français moderne. On y trouve aussi des conseils consacrés à la lecture afin d'assurer une transmission à voix haute respectant sa scansion. C'est tout particulièrement le cas des traductions de Petit de Julleville, de Rœhrich, de Clédat, de Bouchor, de Fabre et de Chamard. Traduire un poème nécessite au préalable d'étudier les qualités ou les caractéristiques qui en font justement une œuvre poétique, soit d'étudier sa versification et plus largement son rythme ou sa «musique », et pas seulement d'analyser son lexique, comme si l'on ne traduisait que les mots du texte, non leur agencement particulier fondé sur une structure métrique.

En revanche, Bédier, dans la préface de son édition et traduction, ne dit pas un mot de la prosodie de la Chanson de Roland. Estimant qu'un

125. Comme en témoigne par exemple ce qu'il écrit au même moment à propos de la chanson de geste dans son étude sur «L'esprit de nos romans de chevalerie » (Revue de France, 1 [1921], p. 88-108) : «Il est beau qu'[elle] nous serve encore, après tant de siècles, à fortifier en nous le sentiment national, et qu'elle nous offre, toujours vivace et expressive, La Légende Dorée de la patrie. Opus francigenum » (p. 108, cité d'après A. Corbellari, Joseph Bédier..., p. 486). Sur le nationalisme de Bédier, voir aussi M. R. WARREN, Creole Medievalism. Colonial France and Joseph Bédier's Middle Ages, Minneapolis, 2011.

126. BoÈce, Traité de la musique, I, 186, éd. et trad. C. MeYer, Turnhout, 2004, p. 30-31. 
traducteur ne peut que détruire la musique d'une œuvre poétique, il se concentre principalement sur sa syntaxe et son lexique. Le style paraît se limiter désormais aux tournures et aux mots du texte. La Chanson de Roland apparaît ainsi comme une œuvre littéraire dont la valeur est fondée avant tout sur la qualité de sa langue, non sur la pulsation rythmique de son chant. Bédier entend l'arracher du même coup aux séductions d'une poésie populaire et d'un chant de guerre auxquels elle était le plus souvent identifiée jusque-là et auxquels semblaient tenir sa valeur et son intérêt, pour la faire entrer dans le canon de la littérature française. Plutôt que d'en proposer une « édition classique » destinée à l'éducation de la jeunesse nationale dans les classes des établissements scolaires français, il en a fait une œuvre «classique » au sens canonique du terme. La fin du chant ne pouvait que coïncider ici avec la fin du combat.

Christopher Lucken - Université Paris 8 (Vincennes/Saint-Denis), EA 7322/ Université de Genève

\section{Traduire la chanson de Roland}

Cette étude présente et analyse pour l'essentiel les nombreuses traductions rythmées ou versifiées de la Chanson de Roland réalisées entre la publication du manuscrit d'Oxford en 1837 et la fin de la Première Guerre mondiale, traductions qui s'efforcèrent de répondre au titre qui avait été donné à cette chanson de geste, d'en restituer d'une manière ou d'une autre le caractère lyrique et de maintenir ainsi le lien que l'on avait établi entre elle et la cantilena Rolandi qui aurait été chantée lors de la bataille de Hastings afin d'encourager les soldats à se battre. Après avoir cherché à préciser ce qui a amené ses premiers traducteurs à en conserver le chant (dans un contexte dominé principalement par la défaite de 1870 et un désir de revanche), elle présente également les arguments avancés en 1922 par Joseph Bédier pour la traduire en prose, plutôt qu'en vers, et s'efforce d'expliquer les motivations et les enjeux d'une telle décision.

cantilena Rolandi, chanson de geste, Chanson de Roland, Joseph Bédier, nationalisme, poésie épique, prose, traduction, vers

\section{Translating The Song of Roland}

This study presents and analyzes the many versified or rhythmic translations of The Song of Roland produced between the publication of the Oxford manuscript in 1837 and the end of the First World War. These translations attempted to keep in mind the title that had been given to this chanson de geste, preserving in one way or another its lyrical character and thus maintaining the link that had been established with the Cantilena Rolandi that was said to have been sung during the Battle of Hastings to encourage soldiers to fight. After having specified what led its first translators to retain the song element of this poem (in a context dominated mainly by the defeat of 1870 and a desire for revenge), it presents the arguments made in 1922 by Joseph Bédier to translate it into prose, rather than verse, and attempts to explain the motives and issues of such a decision.

Cantilena Rolandi, chanson de geste, epic poetry, Joseph Bédier, nationalism, prose, Song of Roland, translation, verse 